\title{
A STUDY OF HOSPITAL ASSOCIATED MENINGITIS IN A NEONATAL UNIT OF A TERTIARY CARE HOSPITAL IN DELHI, INDIA
}

\author{
K. Gupta ${ }^{1}$, V.S. Randhawa ${ }^{1}$, A. Saili ${ }^{2}$, A. Kumar ${ }^{2}$, S. Khare ${ }^{3}$, R. Dutta ${ }^{1}$ \\ ${ }^{1}$ Microbiology, ${ }^{2}$ Pediatrics, Lady Hardinge Medical College, ${ }^{3}$ Microbiology, National Centre for Disease \\ Control, Delhi, India
}

Background and aims: Limited data on epidemiological profile of hospital associated meningitis (HA-M) in neonates in India led to the present one year prospective study at Lady Hardinge Medical College and Smt.Sucheta Kriplani and Kalawati Saran Children's Hospitals, Delhi.

Methods: The key inclusion criteria were,

(1) age: 0-28 days,

(11) stay beyond 48 hours of admission, and

(111) absence of congenital malformations. The diagnosis was based on CDC guidelines. Blood culture was performed where indicated. Inoculation in Hep-2 cell lines was done for enterovirus isolation. Serological tests for coxsackievirus B1-6 and parvovirus B-95 were undertaken. Antimicrobial susceptibility testing was done using CLSI guidelines. More than twenty risk factors were studied and analyzed using EpiInfo software.

Results: Out of 591 neonates enrolled, 32 episodes of HA-M were reported with an incidence of 5.41 HA-M per 100 admissions. 34.4\% cases of HA-M (i.e. highest incidence) occurred in March. Approximately $59.4 \%$ meningitis occurred on day $3 / 4$ of admission. The commonest clinical signs were lethargy and irritability. Approximately $65.6 \%$ of the cases were culture positive. Escherichia coli was the most commonly implicated pathogen. Other key pathogens isolated were Klebsiella spp. and Staphylococcus aureus. The antimicrobial susceptibility pattern varied. Viral etiology could not be established. Birth weight $<2500 \mathrm{gms}$, resuscitation by intubation at birth and total parenteral nutrition were significant risk factors.

Conclusions: Infection control practices need to be strictly enforced to reduce the current incidence of HA$\mathrm{M}$ in the neonatal unit. 